\begin{tabular}{|c|l|}
\hline Title & Morphology and rheology of an immiscible polymer blend subjected to a step electric field under shear flow \\
\hline Author(s) & Orihara, H.; Nishimoto, Y.; A ida, K.; Na, Y H; Nagaya, T.; Ujiie, S. \\
\hline Citation & $\begin{array}{l}\text { Journal of Physics: Condensed Matter, 23/28), 284106 } \\
\text { https://doi.org/L0.1088/0953 8984/23/28/284106 }\end{array}$ \\
\hline Issue Date & 2011 \\
\hline Doc URL & http://hdl.handle.net/2115/51078 \\
\hline Rights & Copyright @2011 IOP Publishing Ltd. \\
\hline Type & article (author version) \\
\hline File Information & orihara 1.pdf \\
\hline
\end{tabular}

Instructions for use 


\title{
Morphology and rheology of an immiscible polymer blend subjected to a step electric field under shear flow
}

\author{
H. Orihara ${ }^{1}$, Y. Nishimoto ${ }^{1}$, K. Aida $^{1}$, Y. H. Na ${ }^{1}$, T. Nagaya $^{2}$ \\ and S. Ujiie ${ }^{3}$ \\ ${ }^{1}$ Division of Applied Physics, Faculty of Engineering, Hokkaido University, Sapporo, \\ Hokkaido 060-8628, Japan \\ ${ }^{2}$ Department of Electrical and Electronic Engineering, Faculty of Engineering, Oita \\ University, Oita 870-1192, Japan \\ ${ }^{3}$ Department of Applied Chemistry, Faculty of Engineering, Oita University, Oita \\ 870-1192, Japan \\ E-mail: orihara@eng.hokudai.ac.jp
}

\begin{abstract}
We have investigated the structural change of an immiscible polymer blend in shear flow when subjected to a step electric field. Three-dimensional images during the process were successfully constructed with a confocal scanning laser microscope and at the same time the transient shear stress was also measured. The interface tensor was calculated from the images. Several factors are incorporated into the shear stress, such as the bulk viscosity, the interfacial tension, and the Maxwell stress. We performed an experiment to separate the Maxwell stress from the total shear stress. The results are discussed in terms of the interface tensor.
\end{abstract}




\section{Introduction}

Immiscible fluid blends (emulsions) exhibit many interesting characteristics in rheology, which are closely related to changes in morphology. When subjected to a flow, these blends undergo changes through complex processes such as deformation, breakup and coalescence. The recent theoretical and experimental successes in understanding the relationships are reviewed by Tucker and Moldenaers [1]. In 1991, Doi and Ohta [2] developed a semiphenomenological theory based on the interface tensor $q_{\alpha \beta}$, in terms of which the excess stress due to the interfacial tension is expressed as $-\Gamma q_{\alpha \beta}[3,4,5]$, where $\Gamma$ is the interfacial tension. Recently, Takahashi et al. [6] made a detailed experimental evaluation. They measured the shear stress and observed the shape change of droplets separately after applying large step strains, from which they found good agreement between the measured shear stress and that calculated from the shape using the above expression. In addition to the interfacial tension, there is another contribution to the shear stress due to the velocity field at the interface and the viscosity difference between two fluids $[3,7,8]$.

In addition to the flow, an electric field is also another effective field for bringing about morphological changes in blends. In fact, under the application of an electric field remarkable changes were reported when the two fluids had mismatched electrical properties, such as permittivity and conductivity $[9,10,11,12,13]$. At the same time, the apparent viscosity increased. This phenomenon is called the electrorheological (ER) effect. In the absence of an electric field, droplets of one fluid are dispersed in the matrix of the other. When subjected to the electric field, the droplets become elongated along the field due to the electric force exerted on the interfaces; sometimes a network structure forms, resulting in an apparent increase in viscosity $[14,15,16]$. The transient response of shear stress to a step electric field was investigated in detail and scaling properties were found [17]. For example, when ER blends are subjected to a step electric field at a constant shear rate, the transient apparent viscosity depends only on the strain, as long as $\dot{\gamma} / E^{2}$ is constant, where $\dot{\gamma}$ and $E$ are the shear rate and electric field, respectively.

In this paper, we present three-dimensional images of a blend that is subjected to a step electric field under steady shear flow and the transient shear stress measured at the same time. The interface tensor is calculated from the images, on the basis of which the relationship between the structure and the shear stress is discussed.

\section{Experiments}

In the experiments, we used a liquid crystalline polymer (LCP) (Fig.1) [10] and a polyisobutylene (PIB). The molecular weight of LCP is 5300. The used PIB is a mixture of two PIBs with different molecular weights of 1400 and 500, the ratio of which is $3: 7$ in weight. All the experiments were done at $25^{\circ} \mathrm{C}$, where LCP is in the isotropic phase. The mesogens play a role in enhancing LCP's permittivity and conductivity. The physical properties are summarized in Table 1. We used PIB because it is a good refractive 


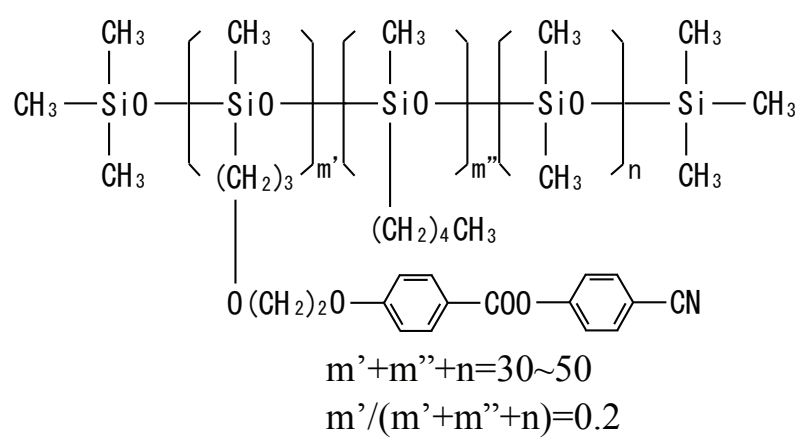

Figure 1. Liquid crystalline polymer (LCP).

Table 1. Physical properties of LCP and PIB.

\begin{tabular}{lllll}
\hline & Viscosity $(\mathrm{Pa} \mathrm{s})$ & Permittivity & Conductivity $\left(\Omega^{-1} \mathrm{~m}^{-1}\right)$ & Refractive index \\
\hline LCP & 65 & 15 & $8.1 \times 10^{-9}$ & 1.488 \\
\hline PIB & 20 & 2.5 & $3.3 \times 10^{-11}$ & 1.490 \\
\hline
\end{tabular}

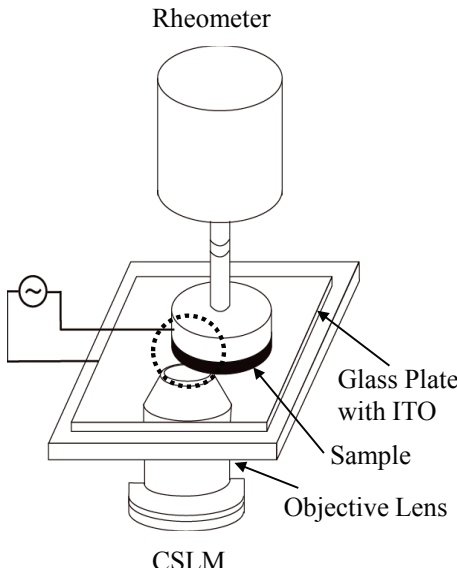

(a)

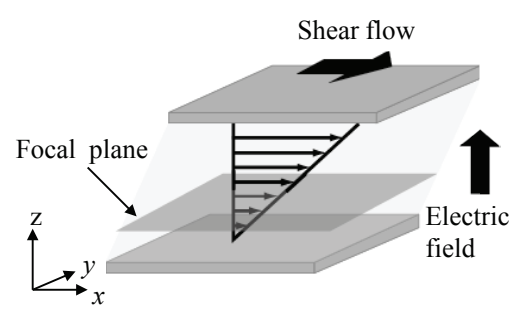

(b)

Figure 2. (a) Schematic illustration of the system combining a CSLM and a rheometer and (b) the details of the sample space.

index-matching fluid with LCP; as shown in Table 1. LCP has greater permittivity and conductivity than PIB. To observe the structure clearly and to distinguish between LCP and PIB, a small amount of fluorescent dye, IANBD amide (Molecular Probes), was doped into LCP before LCP and PIB were mixed. It was confirmed that the dye did not dissolve in PIB. The mixture had the volume ratio of $\mathrm{LCP}: \mathrm{PIB}=1: 8$. In this blend, LCP was dispersed in PIB as droplets in the absence of an electric field.

A schematic picture of our system is shown in Fig. 2 (a). The sample was sandwiched between a bottom glass plate with an ITO (indium tin oxide)-coated electrode and the rotating plate of a rheometer (MCR301, Anton Paar). The diameter 
of the rotating plate was $35 \mathrm{~mm}$, and the gap between the plates is $200 \mu \mathrm{m}$. The shear rate was defined as the one at the edge of the rotating disk and the shear stress there was calculated from the measured torque by assuming that the fluid is Newtonian because a parallel-plate rheometer was used in the experiment. Although immiscible polymer blends under electric fields are not generally Newtonian [18], the above assumption may not seriously affect our conclusions in this paper because the contribution to the stress around the edge is dominant and we will make only a rough theoretical estimation of the shear stress. The structural observation was performed through the bottom glass plate with a confocal scanning laser microscope (CSLM) (IX71, Olympus and CSU22, Yokogawa). The CSLM observation was made $1 \mathrm{~mm}$ from the edge of the rotating disk, meaning that the shear rate at the observation area was almost the same as the value defined above. The two-dimensional (2D) image area is $163 \times 163 \mu \mathrm{m}^{2}$. We carried out both structural observation and rheological measurement at the same time and discussed the relationship.

To construct three-dimensional (3D) images, we used a piezo-actuator (P-721.10, PI) with which the objective lens or the focal plane could move vertically, as shown in Fig. 2 (b) [19], where the $x, y$ and $z$ axes are taken along the flow, vorticity and velocity gradient (the electric field) directions, respectively. We applied a triangular voltage of $5 \mathrm{~Hz}$ to the piezo-actuator so that the objective lens periodically moved up and down between the glass surface and $50 \mu \mathrm{m}$ above it. We used a water-immersion objective lens $(\times 40)$ (the gap between the lens and the bottom glass plate was filled with water) so that the real height of $3 \mathrm{D}$ images was about $56 \mu \mathrm{m}$. The frame rate was $500 \mathrm{fps}$ so that 50 images were captured per half period $(0.1 \mathrm{~s})$, from which one 3D image was constructed. In our experiment the shear rate was $1 \mathrm{~s}^{-1}$, which brought about a total strain of $10 \%$ during the capture of one 3D image. This strain is not sufficiently small, so we made a correction. We assumed that all the points in the blend moved according to an ideal simple shear strain and moved the points back by the distances calculated from the shear rate, and we used 2D images captured only when the objective lens was moving down [20].

Before each measurement, we applied a preshear of $1 \mathrm{~s}^{-1}$ for 3 hours so that the blend became steady under the constant shear. Then we applied a step ac electric field to the blend under steady shear flow. The electric field was supplied by a synthesizer (Multifunction Synthesizer 1940, NF Electric Instruments) and a high voltage amplifier (Model 609C-6, Trek). We used ac electric fields of $1000 \mathrm{~Hz}$ to avoid the conductivity effect.

\section{Results and Discussion}

First, we present the transient shear stress in Fig. 3 when subjected to an ac electric field with an amplitude of $6 \mathrm{kV} / \mathrm{mm}$ at a constant shear rate of $1 \mathrm{~s}^{-1}$. The shear stress increases steeply immediately after the electric field is applied at $t=0$; it takes the maximum and the minimum, then gradually increases until reaching a steady value. 


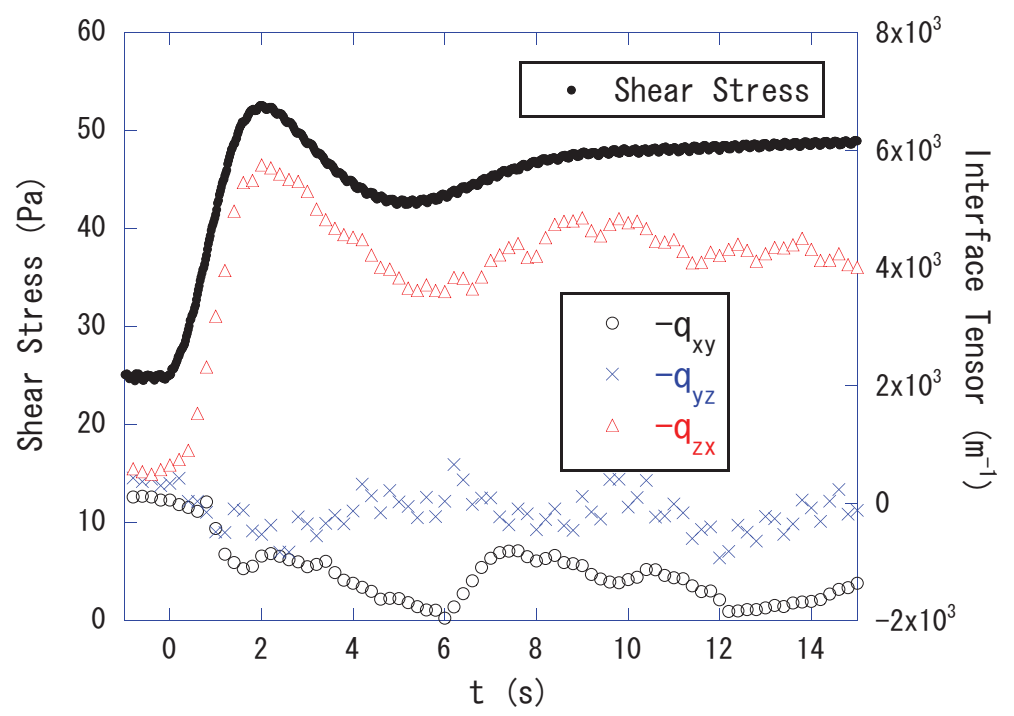

Figure 3. Transient shear stress after applying a step electric field. The off-diagonal elements of the interface tensor are also shown.
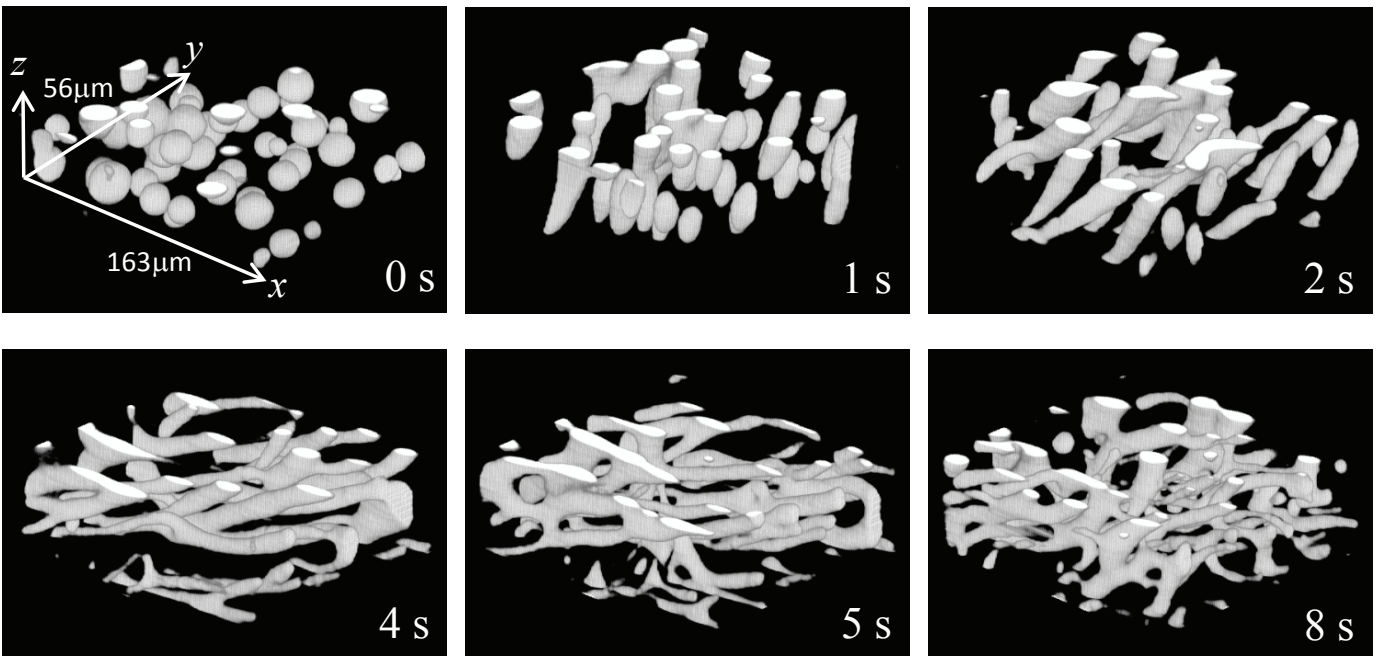

Figure 4. 3D images in the transient process, where the $x, y$ and $z$ axes are taken along the flow, vorticity and velocity gradient (the electric field) directions, respectively.

The transient 3D images captured at the same time as the stress measurement are shown in Fig. 4. The bright parts are LCP, into which a small amount of fluorescent dye was doped.Without the electric field LCP is dispersed as droplets in the PIB matrix and the droplets slide over each other as they flow $(t=0 \mathrm{~s})$. Immediately after application of the electric field all the droplets become elongated along the electric field and some droplets coalesce with each other to form columns $(1 \mathrm{~s})$. The same behavior has been observed without shear flow, and it has turned out that the elongation and coalescence are, respectively, due to the Maxwell stress exerted on the interface and the induced dipoledipole interaction between droplets [19]. Then, the columns gradually tilt toward the 


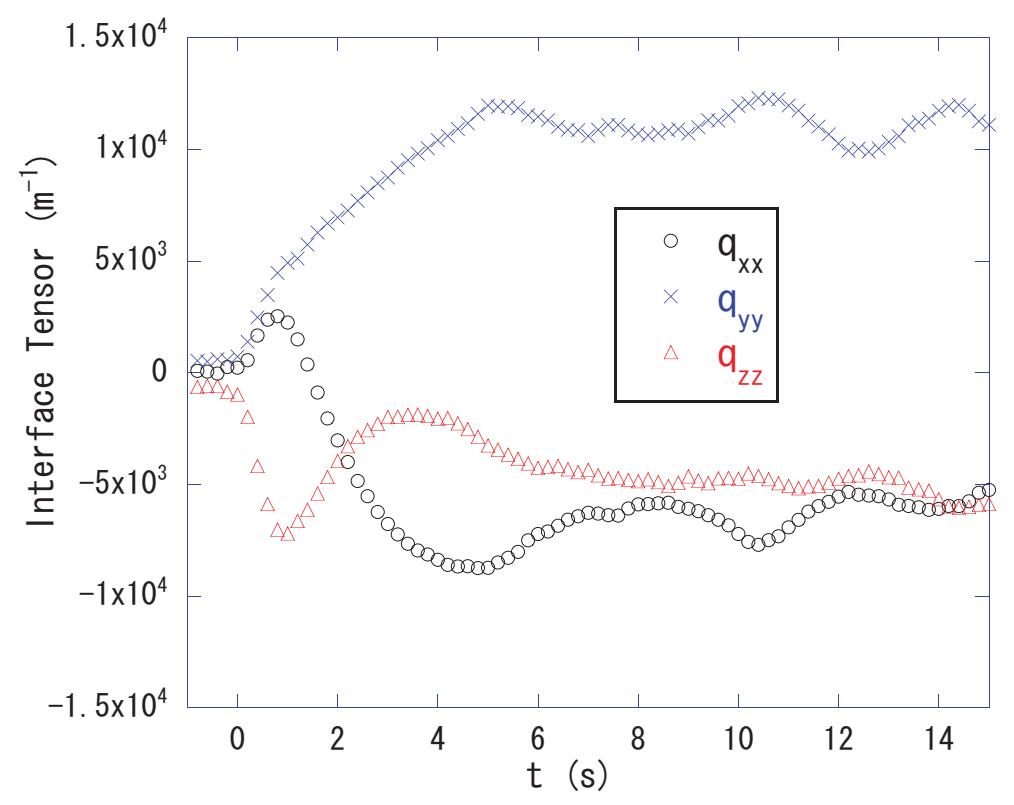

Figure 5. Time dependences of the diagonal elements of the interface tensor.

flow direction and at $2 \mathrm{~s}$ the tilt angle of the columns becomes about $45^{\circ}$, corresponding to the maximum of the shear stress. The columns tilt more (4 s), causing the shear stress to decrease. These results indicate that the elongation and the tilt should be responsible for the shear stress change and that the stress peaks when the tilt angle is $45^{\circ}$. After $4 \mathrm{~s}$, the columns link with each other to form a network structure $(5,8 \mathrm{~s})$, giving rise to the stress increase again as shown in Fig. 3.

Now, let us quantitatively investigate the structural change in terms of the interface tensor, $q_{\alpha \beta}$, defined as

$$
q_{\alpha \beta}=\frac{1}{V} \int_{S}\left(n_{\alpha} n_{\beta}-\frac{1}{3} \delta_{\alpha \beta}\right) d S
$$

where $\mathbf{n}$ is the unit vector normal to the interface, $V$ is the system volume and the integral is done over the whole interface $S$ in the system. The interface tensor is symmetrical and traceless; it expresses the anisotropy and becomes zero for the isotropic structure. For example, with a prolate spheroid elongated along the $z$ axis, $q_{z z}<q_{x x}=q_{y y}$ and $q_{x y}=q_{y z}=q_{z x}=0$. Note that the component along the elongated direction becomes small. Furthermore, when it tilts toward the $x$ direction, the offdiagonal element $q_{x z}=q_{z x}$ becomes nonzero.

Figure 5 shows the time dependences of the diagonal elements corresponding to Fig. 3. In the absence of an electric field, all the components are almost zero because droplets are almost spherical. Immediately after a step electric field is applied, $q_{z z}$ decreases while both $q_{x x}$ and $q_{y y}$ increases, indicating that the droplets become elongated along the electric field (the $z$ axis). Next, $q_{x x}$ decreases and $q_{z z}$ increases, corresponding to the tilting of the elongated droplets due to the shear flow along the $x$ axis. The time dependences of the off-diagonal elements are shown in Fig. 3, where we have plotted 
$-q_{\alpha \beta}$. Both $-q_{x y}$ and $-q_{y z}$ are almost zero, as expected from the symmetry. On the other hand, $-q_{z x}$ increases steeply immediately after application of the electric field; after taking the maximum and the minimum, it then gradually increases until reaching a steady value. This behavior is quite similar to that of the shear stress. In fact, it was shown that the contribution of the interfacial tension to the shear stress is expressed as $-\Gamma q_{\alpha \beta}[3,4,5]$, where $\Gamma$ is the interfacial tension. Here it should be noted that $q_{z x}$ peaks when the principal axis of the surface tensor along the elongated direction is $45^{\circ}$ with respect to the flow direction. This is the reason why the shear stress peaks when the tilt angle of the columns becomes $45^{\circ}$. However, the total shear stress consists of contributing factors other than the interfacial tension as is shown below.

Without an electric field the shear stress for a blend of two Newtonian fluids is approximated as $[3,7]$

$$
\sigma_{\alpha \beta}=-p \delta_{\alpha \beta}+\eta_{2}\left(\frac{\partial v_{\alpha}}{\partial x_{\beta}}+\frac{\partial v_{\beta}}{\partial x_{\alpha}}\right)-\Gamma q_{\alpha \beta}-\frac{\left(\eta_{2}-\eta_{1}\right)}{V} \int_{S}\left(u_{\alpha} n_{\beta}+u_{\beta} n_{\alpha}\right) d S,(2)
$$

where $p$ is the isotropic pressure, $\mathbf{v}$ is the macroscopic velocity, $\eta_{1}$ and $\eta_{2}$ are the viscosities of LCP and PIB, respectively, and $\mathbf{u}$ is the local velocity on the interface. Note that in our experiments $\sigma_{z x}$ was measured. The second term on the right-hand side of Eq. (2) is the viscous stress of the matrix and the third term comes from the interfacial tension mentioned above. The last term is the interface velocity term, which disappears for equal viscosity blends. For the present case, we have to add a contribution from the electric field. Unfortunately, however, we have no theory directly applicable to our system. Under this circumstance we use an expression of the shear stress for a columnar structure, which appeared when a droplet-dispersed structure was subjected to an electric field without shear flow in the same blend. Many LCP columns were formed between the top and bottom plates along the electric field, and the structure was stable for a long time. When the columns were tilted by a shear strain, electric torque appeared to rotate the columns so that they were parallel to the electric field. This torque may originate from the Maxwell stress tensor, $T_{i j}=\varepsilon E_{i} E_{j}-\varepsilon E_{k} E_{k} \delta_{i j} / 2$, where $\varepsilon$ is the dielectric constant. From this tensor the static shear modulus for $\sigma_{z x}$ was calculated as [19]

$$
\begin{aligned}
G(0)= & \pi n a \Gamma+2 \operatorname{nad}\left(\varepsilon_{1}-\varepsilon_{2}\right) E_{0}^{2} \\
& \times \operatorname{Re}\left[\sum_{m=1}^{\infty} \frac{\left\{1-(-1)^{m}\right\}\left(\widetilde{\varepsilon}_{1}-\widetilde{\varepsilon}_{2}\right) / m^{3} \pi^{2}}{\widetilde{\varepsilon}_{1} I_{1}^{\prime}(m \pi a / d) / I_{1}(m \pi a / d)-\widetilde{\varepsilon}_{2} K_{1}^{\prime}(m \pi a / d) / K_{1}(m \pi a / d)^{3}}\right]
\end{aligned}
$$

with

$$
\widetilde{\varepsilon}_{j}=\varepsilon_{j}+\frac{\sigma_{j}}{i \omega} \quad(j=1,2),
$$

where $n$ is the number of columns per unit area, $a$ is the radius of a column, $d$ is the gap between the plates, $\varepsilon_{1}, \varepsilon_{2}, \sigma_{1}$ and $\sigma_{2}$ are the dielectric constants and conductivities of LCP and PIB, respectively; $E_{0}$ and $\omega$ are the amplitude and the angular frequency of the applied electric field, respectively; $I_{1}(x)$ and $K_{1}(x)$ are the modified Bessel functions 


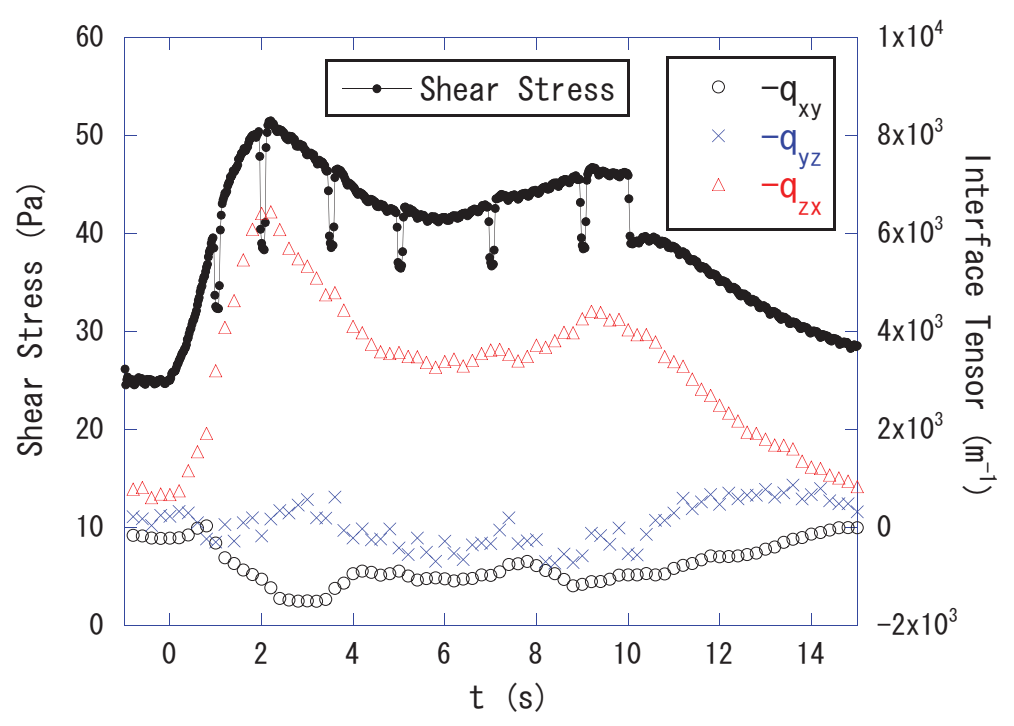

Figure 6. Time dependences of shear stress with intermittent removal of electric field and the corresponding off-diagonal elements of interface tensor.

of the first and second kinds, respectively; and $I_{1}^{\prime}(x)$ and $K_{1}^{\prime}(x)$ are their derivatives. The first term on the right-hand side of Eq. (3) is due to the interfacial tension and the second term originates in the Maxwell stress exerted on the interface. We measured the static shear modulus of the columnar structure with the oscillation method and obtained good agreement between the experiment and the above theory [19]. With the use of the above equations, we will discuss the relationship between structure and shear stress. Before that discussion, however, we present another experimental result, which enables us to separate the electric stress from the total shear stress.

It is easily seen from Eq. (3) that the electric stress (the second term), which we denote as $\sigma_{M}$, should disappear when the electric field is turned off. This gives us a method to separate the electric stress. In the experiment we intermittently removed the electric field for a short time (duration: $0.125 \mathrm{~s}$ ) during a measurement. The result is shown in Figure 6, where the experimental conditions are the same as in Fig. 3 except with this intermittent removal of the electric field and the complete removal after 10 s. The outline of the stress response is almost the same as in Fig. 3 except for the sudden decrease, meaning that the intermittent removal would not affect the structural evolution. The decrease may correspond to the contribution of the Maxwell stress.

First, we examine the stress $\bar{\sigma}_{M}$ that remains after subtracting the electric stress $\sigma_{M}$ from the total stress. $\bar{\sigma}_{M}$ may become $\sigma_{z x}$ in Eq. (2), which includes the bulk stress, interfacial tension and interface velocity terms. Figure 7 shows a plot of $\bar{\sigma}_{M}$ vs the corresponding $q_{z x}$, where we have also plotted the data after completely removing the field. A linear relation is seen for both the intermittent and complete removal of the electric field though the data are scattered for the former. The slopes are 0.0026 and $0.0038 \mathrm{Nm}^{-1}$ for the intermittent and complete removal, respectively, which become the interfacial tension if the surface velocity term is negligible. The difference between the 


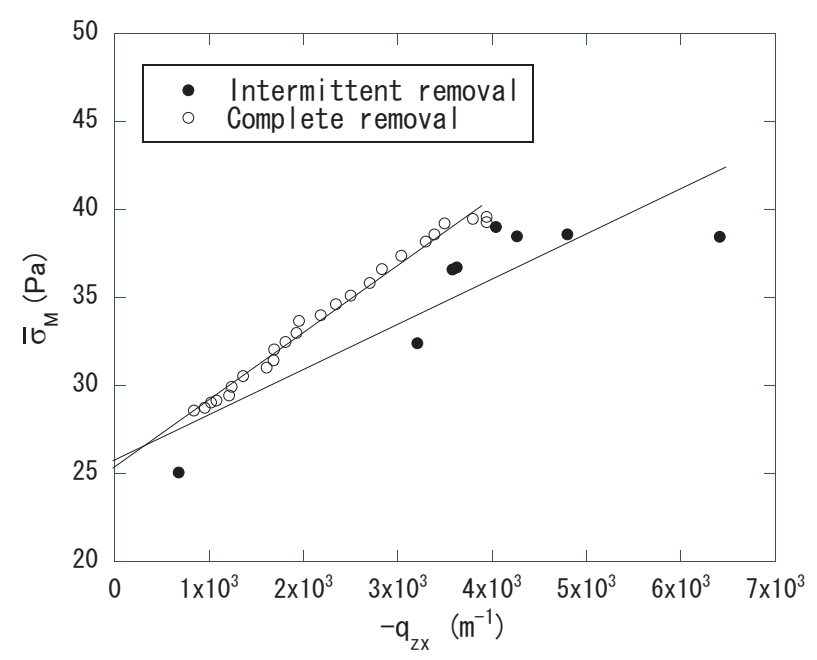

Figure 7. Dependence of $\bar{\sigma}_{M}$ on $q_{z x}$ for intermittent and complete removal.

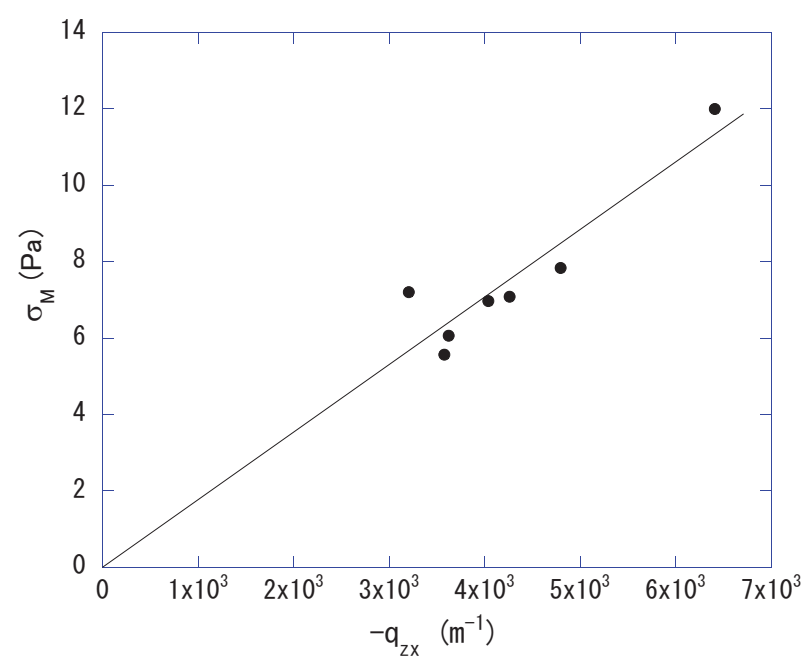

Figure 8. Plot of $\sigma_{M}$ vs $q_{z x}$.

two slopes might come from the surface velocity term. At present, however, we have no information on this term, and therefore we will neglect it hereafter in this paper. Actually, the interfacial tension was determined from the deformed drop retraction method [21] as about $0.003 \mathrm{Nm}^{-1}$, indicating that the surface velosity term may be smaller than the interfacial term. The intercept with the $\bar{\sigma}_{M}$ axis is around 26 Pas, which is nearly equal to the shear stress of PIB with a viscosity of $20 \mathrm{~Pa}$ s under a shear rate of $1 \mathrm{~s}^{-1}$. The above results reveal that the interfacial tension plays an important role in the shear stress change.

Next, let us investigate the Maxwell stress $\sigma_{M}$. From Fig. 6 it seems that $\sigma_{M}$ may be proportional to $\sigma_{z x}$, so we plotted $\sigma_{M}$ vs $q_{z x}$ in Fig. 8. We have a rough proportionality relationship between them. However, we can obtain a better expression by taking into account the dimension and the symmetry. $\sigma_{M}$ may have the following 


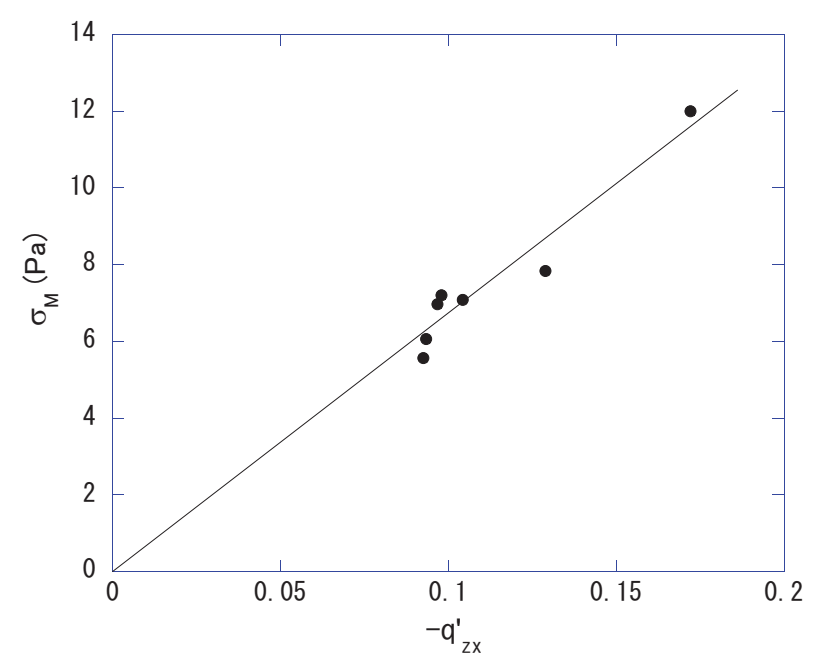

Figure 9. Plot of $\sigma_{M}$ vs $-q_{z x}^{\prime}$.

form:

$$
\sigma_{M}=f\left(\varepsilon_{2} / \varepsilon_{1}\right) \varepsilon_{1} E_{0}^{2}\left(-q_{z x}^{\prime}\right)
$$

where we have assumed that the conduction is negligible because the frequency of the ac elctric field is high enough, $f(x)$ is a function of $\varepsilon_{2} / \varepsilon_{1}$ and $q_{z x}^{\prime}$ is a dimensionless quantity related to $q_{z x}$. Since $q_{z x}$ has the dimension of $\mathrm{m}^{-1}$ we can easily take $q_{z x}^{\prime}$ as $q_{z x} V / S$, where $S$ is the total surface area. As shown in Fig. 9, we have a good proportionality relation, where the proportionality constant $\left(f\left(\varepsilon_{2} / \varepsilon_{1}\right) \varepsilon_{1} E_{0}^{2}\right.$ in Eq. (5)) is about $67 \mathrm{~Pa}$. Hereafter, we will discuss the constant on the basis of Eq. (3), which was originally derived for a columnar structure. For the columnar structure, $q_{z x}$ can easily be calculated as $\pi a n \gamma$, where $\gamma$ is the strain. In addition, $V / S$ is $1 /(2 \pi a n)$, yielding $q_{z x}^{\prime}=\gamma / 2$. As $\sigma_{M}$ is equal to the second term on the right-hand side of Eq. (3) multiplied by $\gamma$, the proportionality constant is given as twice the second term. Furthermore, using the relation $n a d=4 \phi d /(\pi a)$ with the volume fraction of LCP, $\phi$, we obtain

$$
\begin{aligned}
f\left(\varepsilon_{2} / \varepsilon_{1}\right)= & \frac{4 \phi}{\pi} \frac{d}{a}\left(1-\frac{\varepsilon_{2}}{\varepsilon_{1}}\right) \\
& \times\left[\sum_{m=1}^{\infty} \frac{\left\{1-(-1)^{m}\right\}\left(1-\varepsilon_{2} / \varepsilon_{1}\right) / m^{3} \pi^{2}}{I_{1}^{\prime}(m \pi a / d) / I_{1}(m \pi a / d)-\left(\varepsilon_{2} / \varepsilon_{1}\right) K_{1}^{\prime}(m \pi a / d) / K_{1}(m \pi a / d)}\right](\text { ) }
\end{aligned}
$$

where we have assumed that the conduction is negligible. It should be noted that this equation contains the aspect ratio $d / a$. For $d / a=3$, we can obtain $f\left(\varepsilon_{2} / \varepsilon_{1}\right) \varepsilon_{1} E_{0}^{2}=192$ $\mathrm{Pa}$. This is considerably larger than the experimental value, but Eq. (6) may be enough to estimate the order. It should be emphasized that Eq. (6) has been derived for the columnar structure, but it may hold also for the network structure because the proportional relation was confirmed from the experiment. 


\section{Conclusions}

3D images were obtained in the transient process when a polymer blend was subjected to a step electric field under steady shear flow, while at the same time the stress was measured. Furthermore, we performed an experiment to separate the contribution of the Maxwell stress from the total shear stress by intermittently removing the field. The interface tensor was calculated from the images and it was confirmed that the contribution of the interfacial tension is expressed as $-\Gamma q_{z x}$. In addition, the Maxwell stress was found to be proportional to a dimensionless quantity $q_{z x}^{\prime}=q_{z x} V / S$, and the proportionality constant was theoretically estimated. However, it should be noted that the theory we utilized is for static strain and that we did not consider the surface velocity term; it is therefore necessary to modify the theory by taking the flow into account.

\section{Acknowledgments}

This work was supported by a Grant-in-Aid for Scientific Research [Priority Area "Soft Matter Physics' (Contract No. 463). One of the authors (H.O.) would like to thank Prof. K. Yakubo for valuable discussions on the interface tensor.

\section{References}

[1] Tucker III C L and Moldenaers P 2002 Annu. Rev. Fluid Mech. 34177

[2] Doi M and Ohta T 1991 J. Chem. Phys. 951242

[3] Batchelor G K 1970 J. Fluid. Mech. 41545

[4] Onuki A 1987 Phys. Rev. A 355149

[5] Onuki A 1994 Europhys Lett. 28175

[6] Takahashi M, Macaubas P H P, Okamoto K, Jinnai H and Nishikawa Y 2007 Polymer 482371

[7] Mellema J and Willemse W M 1983 Physica A122 286

[8] Okamoto K and Takahashi M 2008 Nihon Reoroji Gakkaishi 3643

[9] Tajiri K, Ohta K, Nagaya T, Orihara H, Ishibashi Y, Doi M and Inoue A 1997 J. Rheol. 41335

[10] Inoue A Ide Y, and Oda H 1997 J. Appl. Polym. Sci. 641319

[11] Kimura H, Aikawa K, Masubuchi Y, Takimoto J, Koyama K and Minagawa K 1998 Rheol. Acta 3754

[12] Pan X and McKinley G 1997 J. Colloid Interface Sci. 195101

[13] Ha J and Yang S 2000 J. Rheol. 44235

[14] Orihara H, Ikeyama Y, Ujiie S and Inoue A 2003 J. Rheol. 471299

[15] Orihara H, Shibuya T, Nagaya T and Ujiie S 2006 J. Phys. Soc. Jpn. 75063802

[16] Na Y H, Aida K, Sakai R, Kakuchi T and Orihara H 2009 Phys. Rev. E 80061803

[17] Orihara H, Tanabu K and Na Y H 2008 J. Phys. Soc. Jpn. 77093802

[18] Orihara H, Taki A, Doi M and Inoue A 2001 J. Rheol. 451479

[19] Aida K, Na Y H, Nagaya T and Orihara H 2009 Phys. Rev. E 80041807

[20] In preparation

[21] Luciani A, Champagne M F and Utracki L A 1997 J. Polym. Sci. B 351393 